\title{
TCEC12: the 12th Top Chess Engine Championship
}

Article

Accepted Version

Haworth, G. and Hernandez, N. (2019) TCEC12: the 12th Top Chess Engine Championship. ICGA Journal, 41 (1). pp. 24-30. ISSN 1389-6911 doi: https://doi.org/10.3233/ICG-190090 Available at https://centaur.reading.ac.uk/76985/

It is advisable to refer to the publisher's version if you intend to cite from the work. See Guidance on citing.

To link to this article DOI: http://dx.doi.org/10.3233/ICG-190090

Publisher: The International Computer Games Association

All outputs in CentAUR are protected by Intellectual Property Rights law, including copyright law. Copyright and IPR is retained by the creators or other copyright holders. Terms and conditions for use of this material are defined in the End User Agreement.

\section{www.reading.ac.uk/centaur}

\section{CentAUR}

Central Archive at the University of Reading

Reading's research outputs online 


\section{TCEC12: the $12^{\text {th }}$ Top Chess Engine Championship}

Guy Haworth and Nelson Hernandez ${ }^{1}$

Reading, UK and Maryland, USA

After the successes of TCEC Season 11 (Haworth and Hernandez, 2018a; TCEC, 2018), the Top Chess Engine Championship moved straight on to Season 12, starting April $18^{\text {th }} 2018$ with the same divisional structure if somewhat evolved.

Five divisions, each of eight engines, played two or more 'DRR' double round robin phases each, with promotions and relegations following. Classic tempi gradually lengthened and the Premier division's top two engines played a 100-game match to determine the Grand Champion. The strategy for the selection of mandated openings was finessed from division to division. The revised TCEC engine lineup is illustrated and listed in Fig. 1 and Table 1.

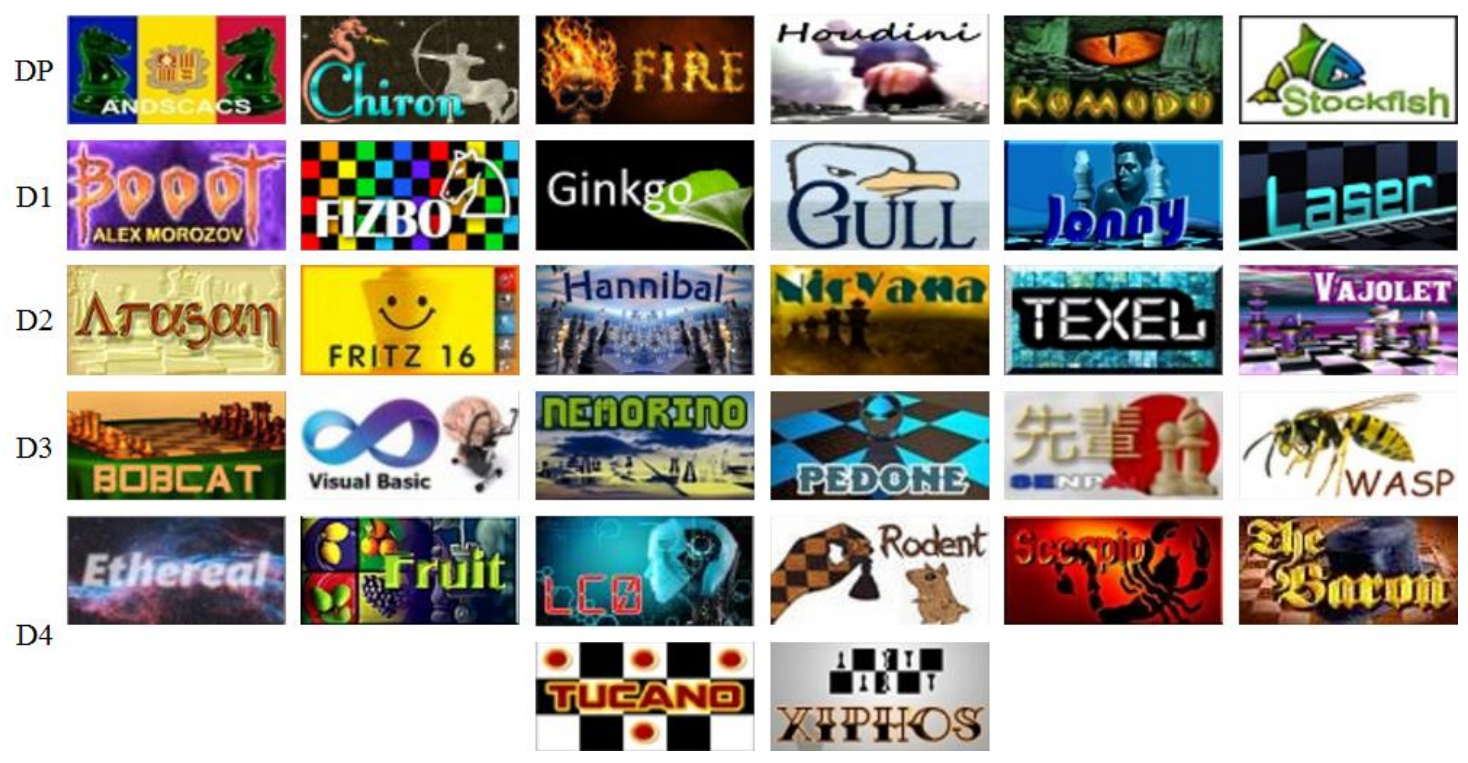

Fig. 1. Logos for the TCEC 12 engines as in their original divisions.

Besides using FIDE's 3x-repetition and 50-move drawing rules, TCEC terminated a game at move 40 or later if both engines had |eval $\mid<0.05$ for ten consecutive plies in the current phase, i.e., since the last pawn-advance and/or capture. TCEC12 fell in line with most of the participating engines by adjudicating endgames using the Syzygy DTZ 50 " EGTs (de Man, 2018) rather than the Gaviota DTM EGTs which do not recognise the 50-move rule. 5-man EGTs were used for the divisions and 6-man EGTs were used for the Superfinal. Games which were apparently decisive were terminated by TCEC

\footnotetext{
${ }^{1}$ Corresponding author: g.haworth@ reading.ac.uk
} 
if both engines consistently agreed for the last eight plies that the evaluation is at least 6.5 or at most 6.5 .

ELO ranged from 2714 to 3554, averaging 3143. Four new engines joined the fray this time:

LEELA CHESS ZERO, a new-architecture UCT/NN engine from a large community, RODENT by the Polish chess programmer Pawel Koziol,

TUCANO by the Brazilian professional software developer/programmer Alcides Schulz, and XIPHOS by the Serbian mathematician and computer scientist Milos Tatarevic.

The formidable 44-core server of TCEC11 was used unchanged in TCEC12.

Table 1. The TCEC12 engines (CPW, 2018), details, authors and progression.

\begin{tabular}{|c|c|c|c|c|c|c|c|c|c|c|c|c|c|}
\hline \multirow{2}{*}{ \# } & \multicolumn{2}{|r|}{ Engine } & \multicolumn{3}{|c|}{ Initial } & \multirow{2}{*}{ thr. } & \multirow{2}{*}{$\begin{array}{l}\text { proto- } \\
\text { col }\end{array}$} & \multirow{2}{*}{$\begin{array}{c}\text { Hash } \\
\text { Kb }\end{array}$} & \multirow{2}{*}{ EGTs } & \multirow{2}{*}{ Authors } & \multirow{2}{*}{$\begin{array}{l}\text { Country } \\
\text { Codes }\end{array}$} & \multirow{2}{*}{\multicolumn{2}{|c|}{$\begin{array}{l}\text { Final } \\
\text { Div. }\end{array}$}} \\
\hline & $\mathbf{a b}$ & Name & Version & ELO & Div. & & & & & & & & \\
\hline 01 & An & Andscacs & 0.93070 & 3339 & $\mathrm{P}$ & 43 & UCI & 16,384 & - & Daniel José Queraltó & $\mathrm{AD}$ & $\rightarrow$ & $\bar{P}$ \\
\hline 02 & $\mathrm{Ar}$ & Arasan & TCEC12 & 3142 & 2 & 43 & UCI & 16,384 & Syz. & Jon Dart & US & $\searrow$ & 3 \\
\hline 03 & $\mathrm{Bc}$ & Bobcat & 8 & 3072 & 3 & 43 & UCI & 16,384 & - & Gunnar Harms & NL & $\rightarrow$ & 3 \\
\hline 04 & Bo & Booot & 6.2 & 3273 & 1 & 16 & UCI & 8,192 & - & Alex Morozov & UA & $\rightarrow$ & 1 \\
\hline 05 & $\mathrm{Cb}$ & Chessbrain VB & 3.67 & 3024 & 3 & 43 & xboard & 1,200 & - & Roger Zuehlsdorf & DE & $\lambda$ & 2 \\
\hline 06 & $\mathrm{Ch}$ & Chiron & 040318 & 3340 & $\mathrm{P}$ & 43 & UCI & 16,384 & Syz. & Ubaldo Andrea Farina & IT & $\searrow$ & 1 \\
\hline 07 & Et & Ethereal & 9.60 & 2985 & 4 & 43 & UCI & 16,384 & - & Andrew Grant & US & $\lambda$ & 3 \\
\hline 08 & $\mathrm{Fi}$ & Fire & 7 & 3393 & $\mathrm{P}$ & 43 & UCI & 16,384 & Syz. & Norman Schmidt & US & $\rightarrow$ & $P$ \\
\hline 09 & $\mathrm{Fz}$ & Fizbo & 2 & 3284 & 1 & 43 & UCI & 16,384 & Syz. & Youri Matiounine & US & $\rightarrow$ & 1 \\
\hline 10 & $\mathrm{Fr}$ & Fritz & 16.10 & 3165 & 2 & 43 & $\mathrm{UCI}$ & 16,384 & Nal? & Vasik Rajlich & CZ/US & $\nearrow$ & 1 \\
\hline 11 & $\mathrm{Ft}$ & Fruit & $2 \mathrm{E}+07$ & 2934 & 4 & 16 & UCI & 8,192 & Syz. & Daniel Mehrmann & $\mathrm{DE}$ & $\rightarrow$ & 4 \\
\hline 12 & $\mathrm{Gi}$ & Ginkgo & 2.012 & 3267 & 1 & 43 & UCI & 16,384 & - & Frank Schneider & DE & $\nearrow$ & $\mathrm{P}$ \\
\hline 13 & $\mathrm{Gu}$ & Gull & 3 & 3217 & 1 & 43 & UCI & 16,384 & Syz. & Vadim Demichev & $\mathrm{RU}$ & $\searrow$ & 2 \\
\hline 14 & $\mathrm{Ha}$ & Hannibal & 121017 & 3193 & 2 & 16 & UCI & & - & Sam Hamilton, Edsel Apostol & US/PH & $\searrow$ & 3 \\
\hline 15 & Ho & Houdini & 6.03 & 3491 & $\mathrm{P}$ & 43 & UCI & 16,384 & Syz. & Robert Houdart & $\mathrm{BE}$ & $\rightarrow$ & \\
\hline 16 & Jo & Jonny & 8.1 & 3252 & 1 & 43 & UCI & 16,384 & Syz. & Johannes Zwanzger & $\mathrm{DE}$ & オ & 1 \\
\hline 17 & Ko & Komodo & 12 & 3466 & $\mathrm{P}$ & 43 & UCI & 16,384 & Syz. & $\begin{array}{c}\text { Don Dailey, Larry Kaufman, } \\
\text { Mark Lefler }\end{array}$ & US & & \\
\hline 18 & $\mathrm{La}$ & Laser & 070518 & 3194 & 1 & 43 & UCI & 16,384 & Syz. & Jeffrey An, Michael An & US & $\rightarrow$ & 1 \\
\hline 19 & $\mathrm{Lc}$ & Leela Chess Zero & 0.7 ID 125 & 2714 & 4 & 43 & UCI & - & - & UCT/NN AI Community & - & $\searrow$ & - \\
\hline 20 & $\mathrm{Ne}$ & Nemorino & 4.13 & 3104 & 3 & 43 & UCI & 16,384 & Syz. & Christian Günther & US & $\rightarrow$ & 3 \\
\hline 21 & $\mathrm{Ni}$ & Nirvana & 2.4 & 3168 & 2 & 16 & UCI & 8,192 & - & Thomas Kolarik & US & $\rightarrow$ & \\
\hline 22 & $\mathrm{Pe}$ & Pedone & 1.8 & 3104 & 3 & 43 & UCI & 16,384 & Syz. & Fabio Gobbato & IT & $\rightarrow$ & 3 \\
\hline 23 & Ro & Rodent III & 0.244 & 3076 & 4 & 16 & UCI & 4,096 & - & Pawel Koziol & PL & $\rightarrow$ & 4 \\
\hline 24 & $\mathrm{Sc}$ & Scorpio & 2.82 & 2831 & 4 & 43 & xboard & 16,384 & - & Daniel Shawul & ET & $\searrow$ & - \\
\hline 25 & $\mathrm{Se}$ & Senpai & 2.0 & 3062 & 3 & 16 & UCI & 16,384 & - & Fabien Letouzey & FR & $\searrow$ & 4 \\
\hline 26 & St & Stockfish & 160518 & 3554 & $\mathrm{P}$ & 43 & UCI & 16,384 & Syz. & $\begin{array}{l}\text { Tord Romstad, Marco Costalba, } \\
\text { Joona Kiiski, Gary Linscott }\end{array}$ & $\begin{array}{l}\mathrm{NO} / \mathrm{IT} / \\
\mathrm{FI} / \mathrm{CA}\end{array}$ & & \\
\hline 27 & $\mathrm{Te}$ & Texel & $1.08 \mathrm{a} 11$ & 3202 & 2 & 43 & UCI & 16,384 & Syz. & Peter Österlund & SE & 入 & 2 \\
\hline 28 & $\mathrm{Th}$ & The Baron & 3.41 & 2840 & 4 & 43 & UCI & & Syz. & Richard Pijl & NL & $\rightarrow$ & 4 \\
\hline 29 & $\mathrm{Tu}$ & Tucano & 7.00 & 2830 & 4 & 43 & xboard & 1,024 & - & Alcides Schulz & $\mathrm{BR}$ & $\rightarrow$ & \\
\hline 30 & $\mathrm{Va}$ & Vajolet 2 & 2.5 & 3119 & 2 & 43 & UCI & 16,384 & Syz. & Marco Belli & IT & $\rightarrow$ & 2 \\
\hline 31 & $\mathrm{Wa}$ & Wasp & 180420 & 2964 & 3 & 43 & UCI & 8,192 & - & John Stanback & US & $\searrow$ & 4 \\
\hline 32 & $\mathrm{Xi}$ & Xiphos & 0.2 & 2986 & 4 & 43 & UCI & 4096 & - & Milos Tatarevic & $\mathrm{RS}$ & ス & 2 \\
\hline
\end{tabular}

\section{Division 4, two DRR phases, 28 rounds, 112 games, tempo $30^{\prime}+10^{\prime \prime} / \mathrm{m}$}

A principal focus was the participation of the exciting LEELA CHESS ZERO, a neural-networkarchitecture engine inspired by the innovations of Deep Mind's ALPHAZERO (Silver et al, 2017). The 14 most common two-move openings in the second author's CATOBASE (Hernandez, 2018) were allocated to rounds 1-7 and 15-21, and with colours reversed for rounds 8-14 and 22-28, see Table 2. 
The results, as played, are as in Table 3 though a rule introduced in TCEC11 required that the participation of SCORPIO be scratched as it had three technical crashes. The seven connection breaks with the server were thought to be caused by deadlock conditions in the engine. These losses in fact made no difference to the final ranking on this occasion. LCO did not in fact progress but will be greatly helped by GPU assistance in a future season. The generic statistical review of TCEC12 results and terminations is given for each phase of TCEC12 in Table 11.

Table 2. CATOBASE's most common two-move openings.

\begin{tabular}{|c|c|c|c|c|c|c|c|c|}
\hline \multirow[t]{2}{*}{ \# } & \multirow{2}{*}{$\begin{array}{l}\text { First four plies } \\
1 . \text { e4 c5 2. Nf3 d6 }\end{array}$} & \multicolumn{2}{|c|}{ Rounds ECO Opening } & \multirow{2}{*}{$\begin{array}{l}\text { TCEC's ECO coding for the eight games } \\
\text { B84, B52, B50, B52; B51, B54, B53, B90 }\end{array}$} & \multicolumn{4}{|c|}{$\begin{array}{l}\text { TCEC12.D4 results } \\
1-01 / 2-1 / 20 \text { 0-1 ignored }\end{array}$} \\
\hline & & $\& 08 \quad \mathrm{~B} 50$ & & & 3 & 2 & 2 & 1 \\
\hline & 1. e4 e5 2. Nf3 Nc6 & $02 \& 09 \quad \mathrm{C} 44$ & King's Pawn Game & C45, C68, C58, C84; C45, C61, C68, C67 & 5 & 1 & 1 & 1 \\
\hline 03 & 1. d4 Nf6 2. c4 e6 & $03 \& 10$ E00 & Queen's Pawn Game & 50, E40, D35, E20; D30, D50, D59, D41 & 4 & 0 & 4 & 0 \\
\hline 04 & 1. e4 e6 $2 . \mathrm{d} 4 \mathrm{~d} 5$ & $04 \& 11 \mathrm{C} 00$ & French Defence & $1, \mathrm{C} 06, \mathrm{C} 06, \mathrm{C} 01 ; \mathrm{C} 11, \mathrm{C} 14, \mathrm{C} 01, \mathrm{C} 01$ & 4 & 1 & 3 & 0 \\
\hline 05 & 1. e4 c5 2. Nf3 Nc6 & $05 \& 12 \quad$ B30 & Sicilian Defence & $330, \mathrm{~B} 30, \mathrm{~B} 31$ & 2 & 4 & 2 & 0 \\
\hline 06 & & $06 \& 13 \mathrm{~B} 12$ & Caro-Kann Defence & & 2 & 4 & 2 & 0 \\
\hline 07 & 1. $\mathrm{d} 4 \mathrm{~d} 52 . \mathrm{c} 4 \mathrm{c} 6$ & $07 \& 14 \mathrm{D} 10$ & Queen's Gambit Declined & 2, D12, D11, D12; D10, D12, D94, D30 & 3 & 2 & 2 & 1 \\
\hline 08 & 2. $\mathrm{c} 4 \mathrm{~g} 6$ & $15 \& 22$ E60 & King's Indian Defence & $5, \mathrm{~B} 38, \mathrm{E} 61$ & 2 & 2 & 3 & 1 \\
\hline 09 & 1. $\mathrm{d} 4 \mathrm{Nf6} 2 . \mathrm{Nf} 3 \mathrm{~d} 5$ & $16 \& 23$ A46 & Queen's Pawn game & 30, D37, D05, D11; D26, D94, D35, D24 & 3 & 2 & 2 & 1 \\
\hline 10 & 1. $\mathrm{d} 4 \mathrm{~d} 52 . \mathrm{c} 4 \mathrm{e} 6$ & $17 \& 24$ D30 & 's Gambit Declined & D50, D50, D35, E51; D30, D50, D30, D53 & 3 & 2 & 2 & 1 \\
\hline & 1. e4 c5 2. Nf3 e6 & $18 \& 25 \quad B 40$ & Sicilian Defence & $\mathrm{B} 33, \mathrm{~B} 45, \mathrm{~B} 45, \mathrm{~B} 22 ; \mathrm{B}$ & 3 & 1 & 4 & 0 \\
\hline 12 & 1. $\mathrm{d} 4 \mathrm{Nf6} 2 . \mathrm{Nf} 3 \mathrm{e} 6$ & $19 \& 26$ A 46 & Queen's Pawn game & E10, E10, D24, E05; D30, E12, D30, A46 & 4 & 1 & 3 & 0 \\
\hline 13 & 1. c4 e5 2. Nc3 Nf6 & $20 \& 27$ A22 & English Opening & $\mathrm{A} 28, \mathrm{~A} 28, \mathrm{~A} 28, \mathrm{~A} 22 ; \mathrm{A} 28, \mathrm{~A} 22, \mathrm{~A} 28, \mathrm{~A} 28$ & 4 & 1 & 2 & 1 \\
\hline 14 & 1. Nf3 Nf6 2. c4 c5 & $21 \& 28$ A 15 & English Opening & $\mathrm{B} 14, \mathrm{~A} 35, \mathrm{~A} 30, \mathrm{~A} 33 ; \mathrm{D} 30, \mathrm{D} 27, \mathrm{~A} 30, \mathrm{~A} 30$ & 1 & 6 & 1 & 0 \\
\hline
\end{tabular}

For division 4, all rounds have four games so game $r . n$ is game $4 r-4+n$ in the pgn files (Haworth and Hernandez, 2018b) and the colour-flipped pairings of engines are 28 games apart. This division had $10.8 \%$ of its wins below the diagonal of the eventual $\mathrm{x}$-table. ETHEREAL alone was much improved, undefeated and a strong first while XIPHOS kept RODENT III in a distant third place, beating it 4-0.

Table 3. The TCEC12 Division 4 cross-table: two DRR phases, 28 rounds, 112 games.

\begin{tabular}{llcccccccccccccc}
\hline$\#$ Engine & ELO & Pts & DRR & SB & nSB & Et & Xi & Ro & Tu & Th & Ft & Sc & Lc & Move \\
\hline 1 & Ethereal 9.60 & 2985 & 23.5 & 2 & 282.25 & 70.56 & & $11====1=$ & 1111 & $===1$ & $11=1$ & 1111 & 1111 & $\nearrow$ \\
2 & Xiphos 0.2 & 2986 & 22.0 & 2 & 253.75 & 63.44 & $00==$ & & 1111 & $=11=$ & $111=$ & $101=$ & 111 & 1111 & $\nearrow$ \\
3 & Rodent III 0.244 & 3076 & 18.0 & 2 & 187.00 & 46.75 & $==0=0000$ & & $1===11=1$ & $=11=$ & $111=$ & 1111 & $\rightarrow$ \\
4 & Tucano 7.00 & 2830 & 13.5 & 2 & 135.25 & 33.81 & 0000 & $=00=0===$ & & $=111$ & $1=01$ & $==00$ & 1111 & $\rightarrow$ \\
5 & The Baron 3.41 & 2840 & 11.5 & 2 & 123.00 & 30.75 & $===0$ & $000=00=0$ & $=000$ & & $=1=1$ & 1001 & $=111$ & $\rightarrow$ \\
6 & Fruit 20180416 & 2934 & 11.0 & 2 & 118.25 & 29.56 & $00=0$ & $010==00=0=10=0=0$ & & $=010$ & 1111 & $\rightarrow$ \\
7 & Scorpio 2.82 & 2831 & 10.5 & 2 & 105.00 & 26.25 & 0000 & 0000 & $000===11$ & 0110 & $=101$ & & $101=$ & $\searrow$ \\
8 & LCZero 0.7 ID125 & 2714 & 02.0 & 2 & 21.50 & 5.38 & 0000 & 0000 & 0000 & 0000 & $=000$ & 0000 & $010=$ & & $\searrow$ \\
\hline
\end{tabular}

\section{Division 3, two DRR phases, 28 rounds, 112 games, tempo $30^{\prime}+10^{\prime \prime} / \mathrm{m}$}

In this division, the same fourteen openings were mandated as for Division 4 and used in the same order. This time however, the colour-reversed game followed immediately rather than 28 games later, giving an earlier balanced view on the bilateral contests. Rounds were therefore of eight games rather than four and were numbered from 1 to 14 , see Table 4.

Draws under the 50-move rule are very rare in TCEC, less than $1 \%$ of terminations. This is because most engines seem to monitor the ply-count, zero their evaluations as they see it reaching 100, and allow TCEC draw-adjudication to take its course. Game 13.1/97, CHESSBRAINVB-WASP, was however a 50m-rule draw: CHESSBRAINVB retained hopes of a win to the end, despite being a pawn down. 
CHESSBRAINVB was a clear winner but the race for the second promotion-spot was close. XIPHOS pulled off its second promotion this season, despite having lost its head-to-head matches with fellowcandidates ETHEREAL and PEDONE. ETHEREAL more than justified its promotion to Division 3.

Table 4. The 14 two-move openings chosen for Division 3.

\begin{tabular}{|c|c|c|c|c|c|c|c|c|c|}
\hline \multirow{2}{*}{\multicolumn{2}{|c|}{$\begin{array}{l}\text { \# First four plies } \\
\text { 01 } 1 . \text { e } 4 \text { c5 } 2 . \text { Nf3 d6 }\end{array}$}} & \multirow{2}{*}{$\begin{array}{c}\text { Rounds } \\
01\end{array}$} & \multirow{2}{*}{$\begin{array}{c}\text { ECO } \\
\text { B50 }\end{array}$} & \multirow{2}{*}{$\begin{array}{l}\text { Opening } \\
\text { Sicilian Defence }\end{array}$} & \multirow{2}{*}{$\begin{array}{l}\text { TCEC's ECO coding for the eight games } \\
\text { B52, B52; B54, B90; B50, B53; B92, B52 }\end{array}$} & \multicolumn{4}{|c|}{$\begin{array}{l}\text { TCEC12.D3 results } \\
1-01 / 2-1 / 2 \text { 0-1 ignored }\end{array}$} \\
\hline & & & & & & 1 & 6 & 1 & 0 \\
\hline $\mathbf{0 2}$ & 1. e4 e5 2. Nf3 Nc6 & 02 & $\mathrm{C} 44$ & King's Pawn Game & C67, C67; C84, C69; C46, C69; C61, C69 & 2 & 2 & 4 & 0 \\
\hline 03 & 1. $\mathrm{d} 4 \mathrm{Nf6}$ 2. c4 e6 & 03 & E00 & Queen's Pawn Game & E12, E12; E14, E10; E10, E10; E10, E00 & 3 & 5 & 0 & 0 \\
\hline 04 & 1. e4 e6 $2 . \mathrm{d} 4 \mathrm{~d} 5$ & 04 & $\mathrm{COO}$ & French Defence & $\mathrm{C} 07, \mathrm{C} 02 ; \mathrm{C} 01, \mathrm{C} 01 ; \mathrm{C} 06, \mathrm{C} 01 ; \mathrm{C} 01, \mathrm{C} 05$ & 4 & 2 & 1 & 1 \\
\hline 05 & 1. e4 c5 2. Nf3 Nc6 & 05 & B30 & Sicilian Defence & В31, В30; В30, В30; В30, В30; В30, В31 & 1 & 5 & 2 & 0 \\
\hline 06 & 1. e 4 c6 $2 . \mathrm{d} 4 \mathrm{~d} 5$ & 06 & B12 & Caro-Kann Defence & B12, B15; В12, В15; B12, B12; B18, В15 & 3 & 3 & 2 & 0 \\
\hline 07 & 1. $\mathrm{d} 4 \mathrm{~d} 52 . \mathrm{c} 4 \mathrm{c} 6$ & 07 & D10 & Queen's Gambit Declined & D10, D11; D11, D10; D12, D11; D11, D12 & 1 & 6 & 1 & 0 \\
\hline 08 & 1. $\mathrm{d} 4 \mathrm{Nf6} 2 . \mathrm{c} 4 \mathrm{~g} 6$ & 08 & E60 & King's Indian Defence & D85, E90; D85, D90; D83, D85; D86, E60 & 2 & 4 & 2 & 0 \\
\hline 09 & 1. $\mathrm{d} 4 \mathrm{Nf6} 2$. Nf3 d5 & 09 & A46 & Queen's Pawn game & A 46, A $46 ; \mathrm{A} 46, \mathrm{~A} 46 ; \mathrm{A} 46, \mathrm{~A} 46 ; \mathrm{A} 46, \mathrm{~A} 46$ & 3 & 5 & 0 & 0 \\
\hline 10 & 1. $\mathrm{d} 4 \mathrm{~d} 52 . \mathrm{c} 4 \mathrm{e} 6$ & 10 & D30 & Queen's Gambit Declined & D30, D30; D31, D53; D30, D30; D30, D30 & 2 & 5 & 1 & 0 \\
\hline & 1. e4 c5 2. Nf3 e6 & 11 & B40 & Sicilian Defence & B40, B40; B40, B40; B40, B40; B40, B40 & 5 & 3 & 0 & 0 \\
\hline & 1. $\mathrm{d} 4 \mathrm{Nf6} 2 . \mathrm{Nf} 3 \mathrm{e} 6$ & 12 & A46 & Queen's Pawn game & $\mathrm{A} 46, \mathrm{~A} 46 ; \mathrm{A} 46, \mathrm{~A} 46 ; \mathrm{A} 46, \mathrm{~A} 46 ; \mathrm{A} 46, \mathrm{~A} 46$ & 1 & 4 & 3 & 0 \\
\hline & 1. $\mathrm{c} 4 \mathrm{e} 5$ 2. Nc3 Nf6 & 13 & A 22 & English Opening & A 22, A $22 ;$ A 22, A $22 ;$ A 22, A $22 ;$ A 22, A 22 & 0 & 6 & 2 & 0 \\
\hline 14 & 1. Nf3 Nf6 2. c4 c5 & 14 & A 15 & English Opening & $\mathrm{A} 05, \mathrm{~A} 05 ; \mathrm{A} 05, \mathrm{~A} 05 ; \mathrm{A} 05, \mathrm{~A} 05 ; \mathrm{A} 05, \mathrm{~A} 05$ & 3 & 5 & 0 & 0 \\
\hline
\end{tabular}

Table 5. The TCEC12 Division 3 cross-table: two DRR phases, 28 rounds, 112 games.

\begin{tabular}{|c|c|c|c|c|c|c|c|c|c|c|c|c|c|c|c|}
\hline \# & Engine & Rtng & Pts & DRR & SB & nSB & Ch & $\mathbf{X i}$ & Pe & Et & Bo & $\mathrm{Ne}$ & $\mathrm{Se}$ & Wa & Move \\
\hline 1 & ChessBrainVB 3.67 & 3024 & 19.0 & 2 & 246.50 & 61.63 & & $=1=0$ & $====$ & $1=1=$ & $11=1$ & $101=$ & $1==1$ & $1==1$ & $\pi$ \\
\hline 2 & Xiphos 0.2 & 3180 & 17.5 & 2 & 220.25 & 55.06 & $=0=1$ & & $===0$ & $===0$ & $==1=$ & $111=$ & $=1=1$ & $1=11$ & $\lambda$ \\
\hline 3 & Pedone 1.8 & 3104 & 16.0 & 2 & 217.50 & 54.38 & $====$ & $===1$ & & $11==$ & $0=10$ & $==01$ & $1===$ & $1===$ & $\rightarrow$ \\
\hline 4 & Ethere & 3243 & 15.5 & 2 & 196.75 & 49.19 & $0=0=$ & $===1$ & $00==$ & & $=1==$ & $1===$ & $=1==$ & $111=$ & $\rightarrow$ \\
\hline 5 & Bobcat 8 & 3072 & 14.5 & 2 & 183.50 & 45.88 & $00=0$ & $==0=$ & $1=01$ & $=0==$ & & $==11$ & $=1=1$ & $10=1$ & $\rightarrow$ \\
\hline 6 & Nemorino 4.13 & 3104 & 12.5 & 2 & 158.00 & 39.50 & $010=$ & $000=$ & $==10$ & $0===$ & $==00$ & & $11==$ & $==11$ & $\rightarrow$ \\
\hline 7 & Senp & 3062 & 9.0 & 2 & 126.75 & 31.69 & $0==0$ & $=0=0$ & $0===$ & $=0==$ & $=0=0$ & $00==$ & & $====$ & $\searrow$ \\
\hline 8 & Wasp 180420 & 2964 & 8.0 & 2 & 111.75 & 27.94 & $0==0$ & $0=00$ & $0===$ & $000=$ & $01=0$ & $==00$ & $====$ & & $\searrow$ \\
\hline
\end{tabular}

\section{Division 2, two DRR phases, 28 rounds, 112 games, tempo $30^{\prime}+10^{\prime \prime} / \mathrm{m}$}

Fourteen of the most frequent 100 two-move openings in CATOBASE were allocated to rounds 1-14 with, again, the colour-reversed games being played immediately.

Table 6. The 14 two-move openings chosen for Division 2.

\begin{tabular}{|c|c|c|c|c|c|c|c|c|c|}
\hline \multirow[t]{2}{*}{ \# } & \multirow{2}{*}{$\frac{\text { First four plies }}{1 . \mathrm{e} 4 \mathrm{e} 52 . \mathrm{Bc} 4 \mathrm{Nf} 6}$} & \multirow{2}{*}{$\begin{array}{c}\text { Rounds } \\
01\end{array}$} & \multirow{2}{*}{$\begin{array}{r}\text { ECO } \\
\text { C24 }\end{array}$} & \multirow{2}{*}{$\begin{array}{l}\text { Opening } \\
\text { Bishop's O.; Berlin Def. }\end{array}$} & \multirow{2}{*}{$\begin{array}{l}\text { TCEC's ECO coding for the eight games } \\
\mathrm{C} 26, \mathrm{C} 26 ; \mathrm{C} 26, \mathrm{C} 26 ; \mathrm{C} 26, \mathrm{C} 26 ; \mathrm{C} 26, \mathrm{C} 26\end{array}$} & \multicolumn{4}{|c|}{$\begin{array}{l}\text { TCEC12.D2 results } \\
1-01 / 2-1 / 20-1 \text { ignored }\end{array}$} \\
\hline & & & & & & 4 & 0 & 1 & 3 \\
\hline 02 & 1. Nf3 Nf6 2. g3 d5 & 02 & A05 & Reti Opening & A05, A05; A05, A05; A05, E09; A05, A05 & 1 & 4 & 3 & 0 \\
\hline & 1. e4 c5 2. Nc3 e6 & 03 & B23 & & 45, B45; B45, B23; B45, B45; B81, B23 & 3 & 4 & 0 & 1 \\
\hline 04 & 1. Nf3 d5 2. c4 e6 & 04 & A09 & Reti Opening & D37, D38; D55, D37; D38, D32; A13, D26 & 1 & 4 & 2 & 1 \\
\hline & 1. $\mathrm{c} 4 \mathrm{e} 52$ & 05 & A22 & English Opening & & 0 & 6 & 2 & 0 \\
\hline 06 & 1. d4 Nf6 2. Nf3 d5 & 06 & A45 & Queen's Pawn Game & & 3 & 5 & 0 & 0 \\
\hline 07 & & 07 & B1 & Caro-Kann defence & & 2 & 6 & 0 & 0 \\
\hline 08 & & 0 & D3 & t Declined & 553 & 2 & 3 & 2 & 1 \\
\hline 09 & Nc6 & 09 & C60 & Ruy Lopez & C61, C58 & 1 & 7 & 0 & 0 \\
\hline 10 & 2. Nf3 g6 & 10 & B27 & Sicilian, Hungarian Var. & В27, В27; В27, В27; В27, В27; В27, В27 & 2 & 4 & 2 & 0 \\
\hline & & 11 & A5 & Old Indian Defence & A53, A55; A54, A53; A53, E90; E90, A53 & 4 & 2 & 1 & 1 \\
\hline & 1. d4 f5 2. g3 Nf6 & 12 & A 81 & Dutch Defence & A81, A90; A92, A81; A92, A81; A81, A81 & 4 & 2 & 1 & 1 \\
\hline 13 & 1. e4 d5 2. exd5 Nf6 & 13 & B01 & Scandinavian Defence & В01, В01; В01, В01; В01, В01; B01, В01 & 3 & 3 & 1 & 1 \\
\hline 14 & 1. e4 Nf6 2. e5 Nd5 & 14 & $\mathrm{~B} 02$ & Alekhine's Defence & В03, В04; В02, В02; В04, В04; В02, В03 & 4 & 3 & 1 & 0 \\
\hline
\end{tabular}


This time, FRITZ and TEXEL won promotion but the newly promoted XIPHOS and CHESSBRAINVB took the next two places. There were 10 technical crashes in the division, and both ARASAN and HANNIBAL were disqualified and relegated for disconnecting from the server: a pity as they had both scored wins against FRITZ. VAJOLET no doubt counted itself lucky to survive.

Table 7. The TCEC12 Division 2 cross-table: two DRR phases, 28 rounds, 112 games.

\begin{tabular}{|c|c|c|c|c|c|c|c|c|c|c|c|c|c|c|c|}
\hline \# & Engine & ELO & Pts & DRR & SB & nSB & Fr & Te & $\mathbf{X i}$ & Ch & $\mathbf{N i}$ & На & Ar & Va & Move \\
\hline 1 & Fritz 16.10 & 3165 & 18.0 & 2 & 242.75 & 60.69 & & $1=01$ & $=11=$ & $1===$ & $==1=$ & $0=11$ & $101=$ & $==1=$ & $\lambda$ \\
\hline 2 & Texel & 3202 & 17.5 & 2 & 227.50 & 56.88 & $0=10$ & & $===1$ & $1==0$ & & $==1=$ & $=111$ & 1011 & $\lambda$ \\
\hline 3 & Xiph & $31 \mathrm{c}$ & 15 & 2 & 206 & 1.50 & $=00=$ & $===0$ & & $=11=$ & & $==1$ & $=0=$ & $1===$ & $\rightarrow$ \\
\hline 4 & JB 3.68 & 3242 & 13. & 2 & 179 & 44.94 & $0===$ & $0==1$ & $=00=$ & & $10=0$ & $100=$ & $=11$ & $11=1$ & $\rightarrow$ \\
\hline 5 & Nirva & 3168 & 13.5 & 2 & 177.25 & 44.31 & $==0=$ & $010=$ & $00=0$ & $01=1$ & & $1=00$ & $11==$ & $==11$ & $\rightarrow$ \\
\hline 6 & Hanni & 3193 & 13.0 & 2 & 180.50 & 45.13 & $1=00$ & $==0=$ & $===0$ & $011=$ & $0=11$ & & $==0=$ & $==10$ & $\rightarrow$ \\
\hline 7 & Arasan TCEC12 & 3142 & 11.0 & 2 & 155.75 & 38.94 & $010=$ & $=000$ & $==1=$ & $1=00$ & $00==$ & $==1=$ & & $=0==$ & $\searrow$ \\
\hline 8 & Vajolet2 2.5 & 3119 & 10.0 & 2 & 141.50 & 35.38 & $==0=$ & 0100 & $0===$ & $00=0$ & $==00$ & $==01$ & $=1==$ & & $\searrow$ \\
\hline
\end{tabular}

\section{Division 1, two DRR phases, 28 rounds, 112 games, tempo $60^{\prime}+10^{\prime \prime} / \mathrm{m}$}

The normalised Sonneborn-Berger scores suggest that this was the most closely-contested division of TCEC12. Eventually, GINKGO and JONNY triumphed though FIZBO and BOOOT kept the result in doubt until almost the end. Thankfully, we did not see another rash of engine-disconnect fails, the one ultimately irrelevant incident being g8.8/64, FRITZ-JONNY. FRITZ as Black had beaten JONNY in their first, g1.7/7, encounter and hung on to its recent promotion: TEXEL did not.

Table 8 . The TCEC12 Division 1 cross-table: two DRR phases, 28 rounds, 112 games.

\begin{tabular}{|c|c|c|c|c|c|c|c|c|c|c|c|c|c|c|c|}
\hline \# & Engine & ELO & Pts & DRR & SB & nSB & Gi & Jo & $\mathbf{F i}$ & Bo & $\mathbf{L a}$ & $\mathbf{F r}$ & Gu & Te & Move \\
\hline 1 & Ginkgo 2.012 & 3267 & 17.0 & 2 & 226.75 & 56.69 & & $==10$ & 0111 & $==0=$ & $=1==$ & $===1$ & $1===$ & $11==$ & $\lambda$ \\
\hline 2 & Jonny 8.1 & 3252 & 17.0 & 2 & 225.25 & 56.31 & $==01$ & & $====$ & $=110$ & $====$ & $0=11$ & $11=1$ & $===1$ & $\lambda$ \\
\hline 3 & Fizbo 2 & 3284 & 15.0 & 2 & 194.75 & 48.69 & 1000 & $====$ & & $=011$ & $==0$ & $==0=$ & $=1==$ & 1111 & $\rightarrow$ \\
\hline 4 & Booot 6.2 & 3273 & 14.5 & 2 & 199.50 & 49.88 & $==1=$ & $=001$ & $=100$ & & $=1==$ & $1==0$ & $01==$ & $===1$ & $\rightarrow$ \\
\hline 5 & Laser 070518 & 3194 & 14.5 & 2 & 198.25 & 49.56 & $=0==$ & $====$ & $===1$ & $=0==$ & & $=1==$ & $=01=$ & $=101$ & $\rightarrow$ \\
\hline 6 & Fritz 16.10 & 3294 & 13.5 & 2 & 185.75 & 46.44 & $==0$ & $1=00$ & $==1=$ & $0==1$ & $=0==$ & & $1===$ & $=0=1$ & $\rightarrow$ \\
\hline 7 & Gull 3 & 3217 & 11.0 & 2 & 153.75 & 38.44 & $0===$ & $00=0$ & $=0==$ & $10==$ & $=10=$ & $0===$ & & $====$ & $\searrow$ \\
\hline 8 & Texel 1.08a11 & 3273 & 9.5 & 2 & 135.00 & 33.75 & $00==$ & $===0$ & 0000 & $==0$ & $=010$ & $=1=0$ & $====$ & & $\searrow$ \\
\hline
\end{tabular}

\section{Division $P$, four DRR phases, 56 rounds, 224 games, tempo 90'+10'/m}

Table 9. The Premier Division cross-table: four DRR phases, 56 rounds, in fact 168 rather than 224 games.

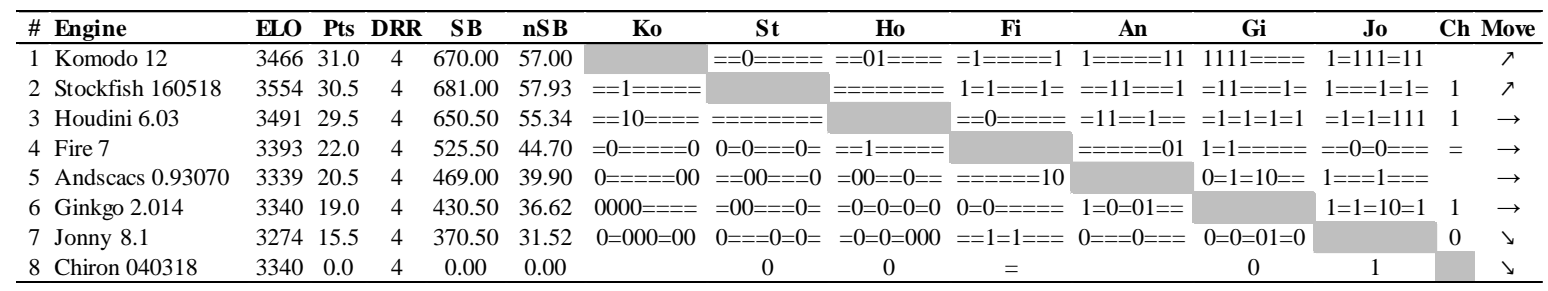

STOCKFISH was the only unbeaten engine but was still second with a Performance ELO of 3443, 121 down on its nominal 3554. KOMODO lost its eight-game match against STOCKFISH but had a better 
harvest of wins against the bottom three engines. HOUDINI made up the three engines that stretched away from the others. CHIRON crashed three times in the first round and was pulled from the event.

\section{The TCEC12 Superfinal match: 100 games, tempo $120^{\prime}+15^{\prime \prime} / \mathrm{m}$}

The Superfinal between STOCKFISH and KOMODO surprised in two ways. First, it was not close and secondly, the win-rate was high. STOCKFISH won 29-9, 23/6 as White and 6/3 as Black so White also dominated Black 29-9. In terms of the fifty two-game pairs, STOCKFISH won 22 pairs $1 \frac{1 / 2-1 / 2}{2}$ and the g71-72 pair 2-0: KOMODO won 4 pairs 11/2-1/2. GM Thechesspuzzler (2018) created a Youtube stream for the TCEC12 Superfinal. Wool (2018) provided some useful chessic insight on TCEC12 as a whole.

Table 10. The Superfinal match of 100 games: (numbers of) games and game-pairs won, Black wins underlined.

\begin{tabular}{|c|c|c|c|c|c|c|}
\hline Superfinal & ELO & Score & \# of & games won (0-1 wins underlined) ... & \# of & game-pairs won \\
\hline STOCKFISH & 3519 & 60 & 29 & $\begin{array}{l}5,9,11,17,25,27,31,35,43, \underline{46}, \\
51,55,57,59, \underline{64}, \underline{66}, 67, \underline{70}, 71, \underline{72}, \\
75,77,83,88,89,93,95,97,99\end{array}$ & 23 & $\begin{array}{l}3,5,6,9,13,14,16,18,22, \\
29,30,32,33,34,35,36, \\
38,39,42,44,47,48,49,50\end{array}$ \\
\hline KOMODO & 3475 & 40 & 9 & $\underline{1}, \underline{29}, 34,38, \underline{45}, 52,56,90,96$ & 4 & $1,15,17,19$ \\
\hline
\end{tabular}

\section{$7 \quad$ Summary}

We gather together some generic statistics for the Divisions and Superfinal in Tables 11 and 12. These will help aficionados and analysts of computer chess to identify the particular games of interest to them later. The pgn files and further data are included with the e-repository version of this note (Haworth and Hernandez, 2018). Our congratulations go once again to the TCEC audience who made for a lively discussion forum (Twitch, 2018), to all participants, particularly to those who gained promotion and to the TCEC12 Grand Champion, STOCKFISH and all its supporters.

Table 11. Generic statistics for each phase of TCEC12.

\begin{tabular}{|c|c|c|c|c|c|c|c|c|c|c|c|c|c|c|}
\hline \multirow{2}{*}{ TCEC 12} & \multicolumn{2}{|c|}{ Division 4} & \multicolumn{2}{|c|}{ Division 3} & \multicolumn{2}{|c|}{ Division 2} & \multicolumn{2}{|c|}{ Division 1} & \multicolumn{2}{|c|}{ Division $\mathbf{P}$} & \multicolumn{2}{|c|}{ Superfinal } & \multicolumn{2}{|c|}{ Overall } \\
\hline & $\#$ & $\%$ & $\#$ & $\%$ & $\#$ & $\%$ & $\#$ & $\%$ & $\#$ & $\%$ & $\#$ & $\%$ & \# & $\%$ \\
\hline \# games & 112 & & 112 & & 112 & & 112 & & 173 & & 100 & & 721 & \\
\hline Draw & 29 & 25.9 & 61 & 54.5 & 53 & 47.3 & 65 & 58.0 & 109 & 63.0 & 62 & 62.0 & 379 & 52.6 \\
\hline Wins & 83 & 74.1 & 51 & 45.5 & 59 & 52.7 & 47 & 42.0 & 64 & 37 & 38 & 8.0 & 342 & 47.4 \\
\hline $1-0$ & 48 & 42.9 & 31 & 27.7 & 35 & 31.3 & 32 & 28.6 & 48 & 27. & 29 & 29.0 & 223 & 30.9 \\
\hline $0-1$ & 35 & 31.1 & 20 & 17.9 & 24 & 21.4 & 15 & 13.4 & 16 & 9.2 & 9 & 9.0 & 119 & 16.5 \\
\hline White Perf. & 62.5 & 55.8 & 61.5 & 54.9 & 61.5 & 54.9 & 64.5 & 57.6 & 102.5 & 59.2 & 60.0 & 60.0 & 412.5 & 57.2 \\
\hline Black Perf. & 49.5 & 44.2 & 50.5 & 45.1 & 50.5 & 45.1 & 47.5 & 42.4 & 70.5 & 40.8 & 40.0 & 40.0 & 308.5 & 42.8 \\
\hline TCEC draw & 13 & 11.6 & 32 & 28.6 & 30 & 26.8 & 38 & 33.9 & 58 & 33.5 & 35 & 35.0 & 206 & 28.6 \\
\hline $3 \mathrm{x}$ repetition & 6 & 5.4 & 10 & 8.9 & 11 & 9.8 & 14 & 12.5 & 25 & 14.5 & 10 & 10.0 & 76 & 10.5 \\
\hline $50 \mathrm{~m}$ rule & 2 & 1.8 & 1 & 0.9 & 0 & 0.0 & 2 & 1.8 & 0 & 0.0 & 1 & 1.0 & 6 & 0.8 \\
\hline EGT a & 11 & 9.8 & 20 & 17.9 & 16 & 14.3 & 13 & 11.6 & 26 & 15.0 & 16 & 16.0 & 102 & 14.1 \\
\hline TCEC win & 71 & 63.4 & 48 & 42.9 & 45 & 40.2 & 44 & 39.3 & 61 & 35.3 & 38 & 38.0 & 307 & 42.6 \\
\hline Tech. default & 7 & 6.3 & 1 & 0.9 & 10 & 8.9 & 1 & 0.9 & 3 & 1.7 & 0 & 0.0 & 22 & 3.1 \\
\hline Manual 'win' & 0 & 0.0 & 0 & 0.0 & 0 & 0.0 & 0 & 0. & 0 & 0. & 0 & 0.0 & 0 & 0.0 \\
\hline Mates & 2 & 1.8 & 0 & 0.0 & 0 & 0.0 & 0 & 0.0 & 0 & 0.0 & 0 & 0.0 & 2 & 0.3 \\
\hline
\end{tabular}


Table 12. The shortest and longest 1-0, drawn and 0-1 games in each phase of TCEC12.

\begin{tabular}{|c|c|c|c|c|c|c|c|c|c|c|c|c|c|c|c|c|c|c|}
\hline \multirow{3}{*}{$\frac{\text { Div. }}{4}$} & \multicolumn{6}{|c|}{$1-0$} & \multicolumn{6}{|c|}{$1 / 2-1 / 2$} & \multicolumn{6}{|c|}{$0-1$} \\
\hline & \multicolumn{3}{|c|}{ Shortest } & \multicolumn{3}{|c|}{ Longest } & \multicolumn{3}{|c|}{ Shortest } & \multicolumn{3}{|c|}{ Longest } & \multicolumn{3}{|c|}{ Shortest } & \multicolumn{3}{|c|}{ Longest } \\
\hline & $\begin{array}{l}\text { Game } \\
16.3 / 63\end{array}$ & 25 & $\mathrm{Xi}-\mathrm{Ft}$ & $\begin{array}{c}\text { Game } \\
18.3 / 71\end{array}$ & 104 & $\mathrm{Ft} \mathrm{C}$ & $\begin{array}{c}\text { Game } \\
21.4 / 84\end{array}$ & 37 & $\mathrm{Xi}-\mathrm{Et}$ & $\begin{array}{l}\text { Game } \\
6.4 / 24\end{array}$ & 353 & $5 \mathrm{C}$ & \begin{tabular}{|c|} 
Game \\
$25.3 / 99$
\end{tabular} & 31 & & $\begin{array}{c}\text { Game } \\
27.3 / 107\end{array}$ & 128 & \\
\hline 2 & & & & & & & & 28 & & & & & & & & & 7 & \\
\hline 1 & & 34 & & & 115 & & & 17 & & & 19 & & & 43 & & & 159 & \\
\hline $\mathbf{P}$ & $1.1 / 1$ & 31 & & $4.3 / 95$ & 99 & Ho-Gi & $40.4 / 160$ & 34 & & $42.3 / 167$ & 185 & $\mathrm{Ko}-\mathrm{Fi}$ & $52.1 / 189$ & 51 & & $2.1 / 5$ & 136 & Jo-Ch \\
\hline
\end{tabular}

\section{REFERENCES}

CPW (2018). https://tinyurl.com/icga046. Biographies of programs and authors.

de Man, R. (2018). http://tablebase.sesse.net/syzygy/. Site providing 5- and 6-man DTZ50" EGTs.

'GM Thechesspuzzler' (2018). https://tinyurl.com/icgaj036. TCEC video playlists.

Haworth, G. $M^{\mathrm{c}} \mathrm{C}$. and Hernandez, N. (2018a). TCEC11: the $11^{\text {th }}$ Top Chess Engine Championship. ICGA Journal 40(3), pp. 216-223. doi: 10.3233/ICG-190081. For supporting data - results, statistics and pgn files, see http://centaur.reading.ac.uk/75899/.

Haworth, G. $M^{\mathrm{c} C}$. and Hernandez, N. (2018b). http://centaur.reading.ac.uk/76985/. TCEC12: the $12^{\text {th }}$ Top Chess Engine Championship. This article plus supporting data - results, statistics and pgn files.

Silver, D. et al (2017) https://arxiv.org/abs/1712.01815 Mastering Chess and Shogi by Self-Play with a General Reinforcement Learning Algorithm.

TCEC (2018) http://tcec.chessdom.com. Current and past TCEC tournaments.

Twitch (2018). https://www.twitch.tv/. A video/chat platform and community for gamers.

Wool, A. (2018) http://mytcecexperience.blogspot.co.uk/ TCEC blog. 